\title{
Topologically inequivalent embeddings
}

\author{
G. B. Mindlin and H. G. Solari \\ Departamento de Física, Facultad de Ciencias Exactas y Naturales, Universidad de Buenos Aires, Pab I, Ciudad Universitaria, \\ Codigo Postal 1428, Buenos Aires, Argentina
}

(Received 27 December 1994)

\begin{abstract}
A chaotic three-dimensional flow can be described through the topological organization of the periodic orbits shadowed by an attractor. If only a scalar variable is available, this description requires the embedding of the data. In this work we report an example in which the reconstructed topology of the flow depends on the chosen embedding.
\end{abstract}

PACS number(s): 05.45.+b

\section{TOPOLOGICAL ANALYSIS OF DATA}

One of the most striking features of nonlinear systems is the possibility of existence of strange attractors. Whenever a natural phenomenon displays a chaotic solution, the repetition of the experiment, even under a well controlled environment, will give rise to outputs that are different if the time series data are directly compared. This observation has guided the research on nonlinear phenomena. How to build up numbers that let us recover the idea of reproducibility, even when long term predictability is lost, has been a key question for the last 15 years.

Recently, a topological approach has been proposed to deal with this issue [1-3]. Shadowed by a strange attractor there is a set of periodic orbits which constitutes its skeleton [4,5]. For three-dimensional (3D) flows (the minimal dimension in which chaos can take place), those periodic orbits constitute closed one-dimensional curves which can be characterized by the way in which they are $k$ notted $[6,7]$. The knot type of a periodic orbit is a topological invariant: as parameters are changed, the orbit might deform and change in shape, but to change its knot type a self-intersection would be necessary, violating determinism (there would be a point with two futures). Other topological invariants can be computed for periodic orbits: if a global Poincare section exists the braid type of the orbit is also an invariant. These ideas will be reviewed in Sec. II.

This approach has been used in the analysis of a number of experimental data sets. It has been proven successful in order to validate models for experimental laser data, fluid data, etc. Many of the data sets analyzed showed that well known geometric mechanisms were responsible for the observed chaotic behavior [8-11].

Yet, the implementation of these ideas is not trivial. Even for reasonably well known systems for which good models exist, the measurement of all the relevant variables might not be technically possible. For example, in the case of the laser with saturable absorber, the measurement of the population inversion is an extremely difficult task (the natural variable to be measured in this problem is the output intensity). In all the examples quoted so far, the problem has been solved by building a threedimensional multivariate environment from the measured scalar data. If the resulting flow has no self-intersections and is smooth, we can think of the reconstructed flow as an embedding of the data.

It is important to realize that despite early hopes, no proof could be found that different good embeddings would give rise to a unique characterization of the flow, and therefore the best that can be said about the system is that one characterizes topologically the pair.data embedding. Actually, it is the purpose of this work to show an example of a parametrically controlled family of embeddings that give rise to nonequivalent topological invariants for the orbits buried within a strange attractor.

This work is organized as follows. In Sec. II, topological invariants are defined for periodic orbits of three dimensional dynamical systems. In Sec. III the model under study is presented. Section IV deals with the problem of finding good embeddings for the flow generated by the model under study, as well as with the topological invariants of the orbits buried within the strange attractor which end up being embedding dependent. Section V contains the discussion of the results and the conclusions.

\section{KNOTS AND BRAIDS}

As mentioned in the previous section, periodic solutions of a 3D dynamical system are closed 1D curves embedded in a $3 \mathrm{D}$ manifold. This is precisely the definition of a knot [13]. If a given closed curve can be deformed into a circle (without self-cuttings) it is called a trivial knot. If that is not the case, it will belong to another class of knots, which will consist of all the closed curves which might be deformed (without self-cuttings) into each other.

The reason for studying knots in the framework of 3D systems is the following. If an orbit is a solution of a dynamical system which belongs to a parametrized fami$1 y$, the control parameters can be changed, and eventually the orbit might disappear. But as discussed in Sec. I as long as the orbit exists, the knot type will not change [7].

A crucial question arises: given a knot, is it possible to compute some number which would indicate to which class it belongs? The question has attracted much research, and the answer is negative. There are a few invariants such that if they are computed for different or- 
bits and give different results, indicate that the knots are not equivalent (not deformable into each other). But the inverse is not true. For every invariant proposed so far in the literature, there are examples of knots which are not equivalent and yet are associated to the same invariant.

Whenever a global Poincáre section exists, a periodic orbit will intersect this section in a finite number of points $n$. One can visualize then a periodic orbit as a set of strands emerging from the $n$ points and terminating in the same set of $n$ points after one excursion to the complement of the Poincaré section in the phase space, i.e., a braid [7].

Two braids are equivalent if we can deform one into the other with the end points fixed and without making a strand cross through another [13]. We shall see now that it is possible to write an algebraic expression for the braid in terms of generators, and that the geometric equivalence restriction mentioned above can be translated into algebraic relationships between the generators. Every braid can be expressed as an ordered set of symbols $\sigma_{1}$, $\sigma_{2}, \ldots, \sigma_{n-1}$ and their inverses $\sigma_{1}^{-1}, \sigma_{2}^{-1}, \ldots, \sigma_{n-1}^{-1}$. Each of the symbols $\sigma_{i}\left(\sigma_{i}^{-1}\right)$ represents a piece of a braid in which the $i$ th strand crosses over (under) the $(i+1)$ th one in a given regular two-dimensional (2D) projection, as displayed in Fig. 1 . Notice that $\sigma_{i} \sigma_{i}^{-1}$ corresponds to two consecutive crossings in which (1) the point $i$ is connected to $i+1$ with a strand that crosses over the one connecting $i+1$ with $i$ and (2) the point $i+1$ is connected back to $i$ with a strand going over the one connecting the point $i$ with the point $i+1$. Observing the resulting braid it is easy to realize that it is possible to deform it by pulling the strand $i \rightarrow i+1 \rightarrow i$ over the strand $i+1 \rightarrow i \rightarrow i+1$. The resulting braid consists of a set of parallel strands with no crossings. Associating this braid to the "identity" $I_{n}$, it is possible to translate our geometric equivalence relationship into the following algebraic one:
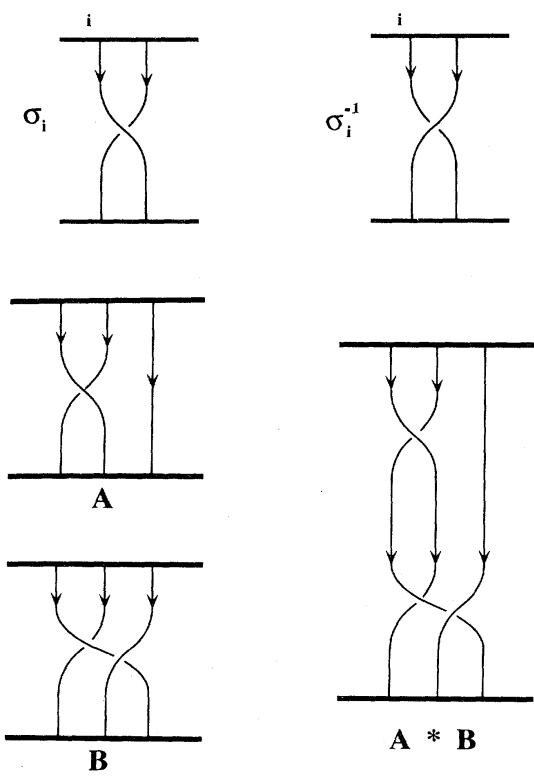

$\mathbf{A} * \mathbf{B}$

FIG. 1. Braid generators (top) and braid product (bottom).

$$
\sigma_{i} \sigma_{i}^{-1}=I_{n} .
$$

There are two algebraic relations that correspond to the geometric moves allowed within a class of braids:

$$
\sigma_{i} \sigma_{i+1} \sigma_{i}=\sigma_{i+1} \sigma_{i} \sigma_{i+1}
$$
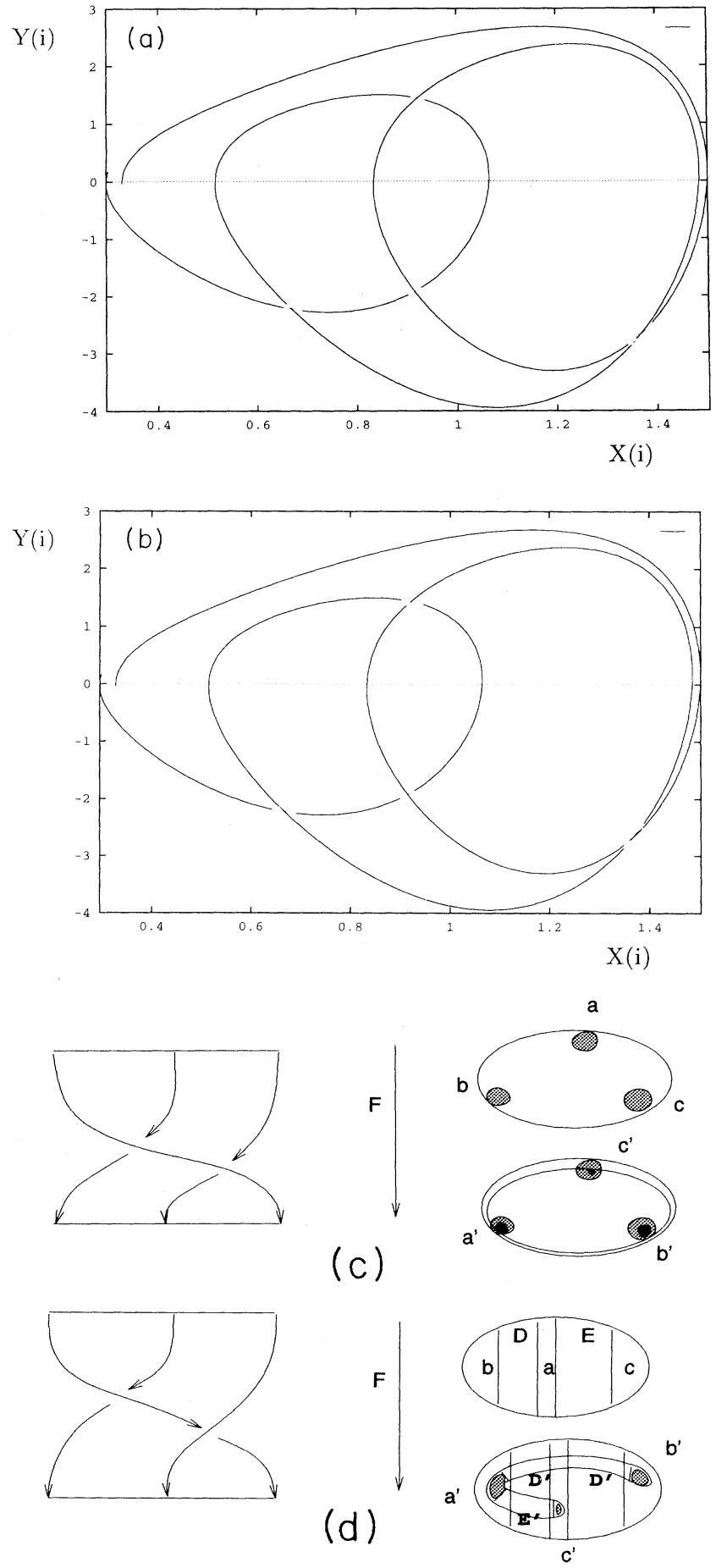

FIG. 2. A well ordered orbit (a), a map induced by the well ordered orbit (b), a badly ordered orbit (c), and a map induced by the badly ordered orbit (d). 


$$
\sigma_{i} \sigma_{j}=\sigma_{j} \sigma_{i},\|i-j\|>1 \text {. }
$$

Notice that two braids corresponding to periodic orbits of the same period do not correspond necessarily to the same class of braids. In Fig. 2, two period three orbits are displayed. The one in Fig. 2(a) (with generators $\left.\sigma_{1}^{-1} \sigma_{2}^{-1}\right)$ can be thought of as a periodic orbit of a rigid rotation of a disk, while the braid in Fig. 2(b) cannot. Let us elaborate this observation. Let us imagine a map that has the first orbit as a solution. For example, let us draw a circle on a Poincaré section such that it encloses the three points in which the periodic orbit crosses the section. Now if we follow the evolution of this circle in the "less complicated way," its image will be a rotated circle [Fig. 2(c)]. The situation is qualitatively different for the second orbit. If we repeat the procedure described above, the image of the initial circle will be a stretched and twisted curve [Fig. 2(d)]. This suggests that a chaotic dynamics might be coexisting with our non rotation compatible braid [14]. The identification of a badly ordered orbit and the statement that its presence implies a chaotic dynamics has been reported in $[3,8]$.

Also notice that both orbits are trivial knots. This observation makes it clear that whenever a global Poincaré section exists, the braid type of an orbit carries more information that its knot type [15].

\section{THE MODEL}

The dynamical system analyzed in this work is a parametrically forced version of the normal form equations for the Takens Bogdanov bifurcation:

$X^{\prime}=Y$,

$Y^{\prime}=\mu[1+\epsilon \cos (\phi)] X+v[1+\epsilon \cos (\phi)] Y-X^{3}+X^{2} Y$,

$\phi^{\prime}=\Omega$.

This study is motivated by the existence of a qualitative agreement between the orbits extracted from a well sampled time series of the experiment reported in [12] and the solutions of the model [16]. The variable $X$ describes the behavior of a symmetry breaking parameter measurable from the pattern, $Y$ stands for the time derivative of that variable, and the forcing term phenomenologically accounts for the dynamics of the effective difference of temperature across the convective cell.

Let us analyze some features of the solutions of the model. If the parameter $\epsilon=0$, the usual Takens Bogdanov scenario is obtained. This consists of the following dynamical behavior as the parameter $\mu_{1}$ is increased, for a fixed negative value of $\mu_{2}$. For $\mu_{1}<0$, a stable fixed point is obtained. For $0<\mu_{1}<-v$, two asymmetric stable fixed points are found which are born in a pitchfork bifurcation from the symmetric fixed point at $(0,0)$. At $\mu=-v$, two Hopf bifurcations from the asymmetric fixed points give rise to periodic limit cycles. The effect of taking $\epsilon<>0$ close to $\mu=-v$ is clear from Fig. 3. As the Hopf frequency is preserved, the flow appears as complex oscillations around oscillations. For the strange attractor displayed in Fig. 3, it is possible to take a global Poincare section consisting of a half plane with the bor-

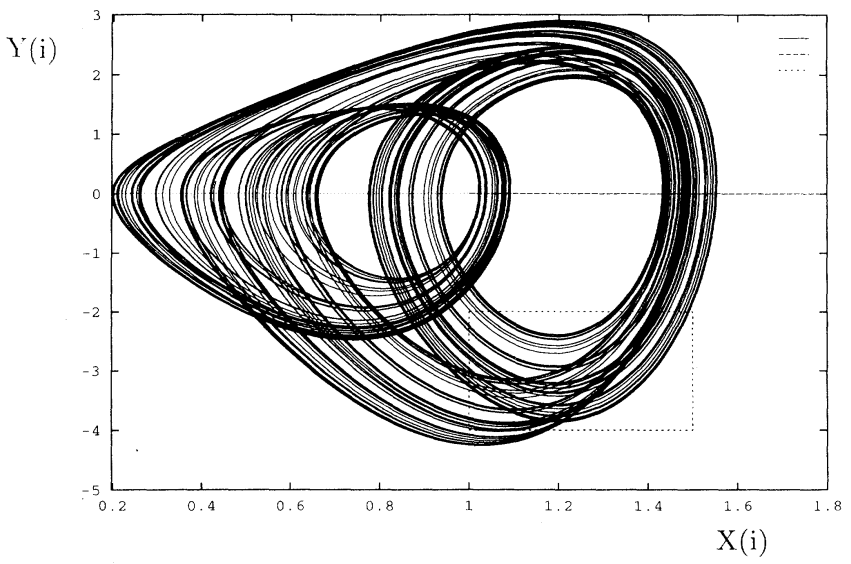

FIG. 3. Strange attractor solution of the numerical model. $X(i)$ vs $Y(i)$ as defined in (4.1) - arbitrary units. The integration was performed with $\mu=1.0434, \epsilon=0.45$, and $\omega=0.399$.

der perpendicular to the plane $(X, Y)$. Notice that this Poincare section is not the stroboscopic section that corresponds to inspect the system at time intervals equal to the forcing period.

A few remarks should be stated at this point. The phase space of this problem is $\mathbb{R}^{2} \times S^{1}$. It is therefore possible to think of the knot properties of the periodic orbits as invariants. Moreover, if we take a stroboscopic map, the braid properties of the orbits can be computed as discussed in Sec. II. Yet, if we take the global Poincaré section indicated in Fig. 3, the characterization of the braids is a subtle issue: being the variable that determines the overcrossings and undercrossings $\phi \in S^{1}$, the rules between generators are different than the ones reported in Sec. II. In any case, the strategy followed in this work consists in taking one variable from the simulations of the system of equations above, and treat this variable as a scalar one. An embedding in $\mathbb{R}^{n}$ will be performed, and we will show that it is possible to embed the data in $\mathbb{R}^{3}$.

\section{EMBEDDINGS AND RECONSTRUCTED FLOWS}

In natural sciences, it is typical to face problems for which even the number of relevant variables is not known. Moreover, once they are discovered, it might not be easy to measure them all. Therefore, it is important to obtain information from a scalar time series data. This has given Whitney's original works a remarkable presence in the last ten years. Whitney proved that a generic smooth map from a $d$-dimensional manifold $M$ to $\mathbb{R}^{2 d+1}$ is a diffeomorphism on $M$ [17]. Takens and Packard built a specific and practical map (the delay-coordinate map) that allows us to construct easily a multivariate environment from scalar data [18]. Recently, Sauer extended these ideas (from manifolds) to compact sets, and (from generic) to probability-one maps $[19,20]$.

The results mentioned above give rise to upper bounds for the dimensionality of the embeddings necessary to embed a manifold or a compact set from their dimen- 
sionality. Another strategy in order to build a multivariate environment that is a good embedding is through the construction of a prescription satisfying the following two features: (1) the map should be one to one, and (2) the map should preserve differential information. The one to one property is conceptually crucial. Our idea that the embedding space is a phase space is based on this property. The state of a deterministic system, and therefore its unique future must be completely specified by a point in the phase space. In other words, no selfintersections should be found in the reconstructed flow.

Following these ideas, we built a code that checks for self-intersections and "cusps" in a 3D flow. Dividing the phase space into boxes, and adjusting their sizes so that just a few points fall within each box (order of 10), the code follows the evolution of those initial conditions. Then, the angles between the speeds at the points are computed. Self-intersections or cusps will be detected whenever large angles appear. This strategy for constructing an embedding follows in spirit the "false neighbors method" [20].

We have analyzed the data file whose $2 \mathrm{D}$ projection is displayed in Fig. 3. A family of parametric 3D embeddings was constructed

$$
\begin{aligned}
X(i) \rightarrow(X(i),[X(i)-X(i-5)], & X(i-\tau)) \\
= & (X(i), Y(i), Z(i))
\end{aligned}
$$

and the number of self-intersections was plotted as a function of $\tau$. The results are summarized in the histogram shown in Fig. 4. Notice that there is an island of values of $\tau, 110<\tau<210$ for which the reconstructed multivariate environment fails to be an embedding, as the flow self-intersects. For $\tau>250$, self-intersections show up again. That leaves us with at least two intervals of $\tau$ such that a three-dimensional embedding for the data is possible for any $\tau$ included in them. Now, a natural question arises: are the two reconstructed flows equivalent?

In order to investigate this issue, close returns in the data were found which approximate unstable periodic or-

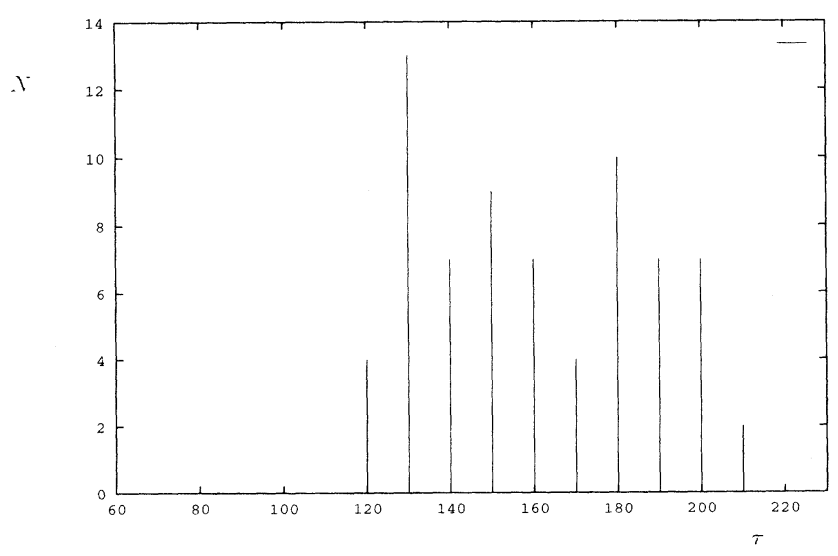

FIG. 4. Number of self-intersections of the flow as $\tau$ is changed. The regions with no self-intersections give rise to deterministic multivariate environments. bits buried within the strange attractor [3]. In Figs. 2(a) and 2(b) the lowest periodic orbit found in the data file under study is shown. In both figures, the diagrams correspond to the projection of the orbits in the $\left(X_{1}, X_{2}\right)$ planes, and the deleted segments indicate the undercrossings. In Fig. 2(a), the diagram corresponds to the orbit embedded with a delay parameter $\tau$ for the third variable that belongs to the first island ( $\tau=60$ ), while the figure in 2(b) corresponds to an embedding parameter corresponding to the second island $(\tau=220)$. Note that all the crossings but one coincide. What does this say about the orbits?

To characterize these two orbits, we can think of them as knots, or we can take advantage of the existence of a global Poincaré section and construct braid invariants. In terms of knot properties, both orbits correspond to trivial knots. In a more precise terminology, the reconstructed orbit displayed in Fig. 2(a) is regular isotopic to the unknot, while the orbit in Fig. 2(b) is ambient isotopic to the unknot. But if we look at the orbits as braids, there is a seemingly dramatic difference between the two orbits. The orbit shown in Fig. 2(b) can be characterized (following the procedures in Sec. II) as a badly ordered orbit $B\left(\sigma_{1}, \sigma_{2}\right)=\sigma_{2}^{-1} \sigma_{1}$, while the one in Fig. 2(a) is a well ordered orbit with $B\left(\sigma_{1}, \sigma_{2}\right)=\sigma_{1}^{-1} \sigma_{2}^{-1}$. Why was there a change in the knot organization as the embedding parameter was changed? Is there a contradiction in this result?

In order to address the first question we will refer to Fig. 5. This figure shows an enlargement of a reconstructed flow, for a delay parameter $\tau=130$. Notice that according to the histogram shown in Fig. 4, selfintersections for the reconstructed flow are found. The enlargement corresponds to the region of the reconstructed space where those intersections take place. In Fig. 3, a square frame indicates its position with respect to the total attractor. Now it is possible to understand why the reconstructed orbits changed their braid types. The flow rests mainly in a three branched $2 \mathrm{D}$ manifold, and for different values of the embeddings, one branch crosses over another one.

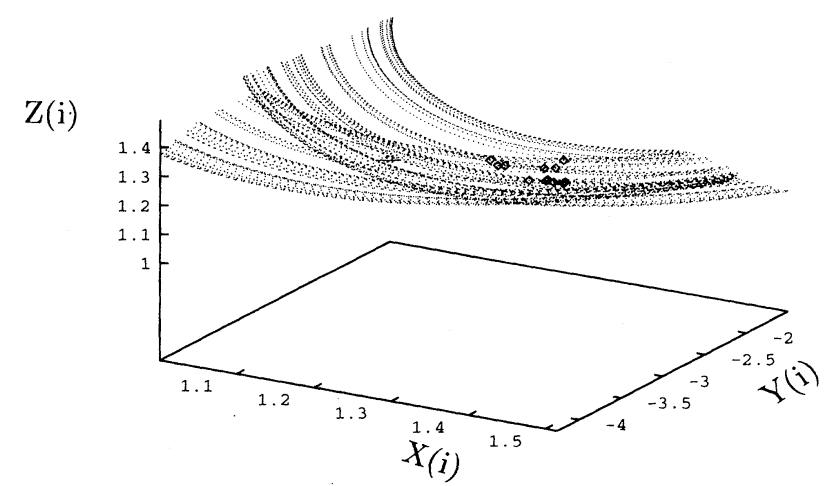

FIG. 5. One branch of the attractor crossing through another one when a $\tau$ was used in order to build the multivariate environment such that self-intersections existed $(\tau=130)$. The graph corresponds to $X(i), Y(i), Z(i)$ as defined in (4.1). The dark points indicate their locations. 


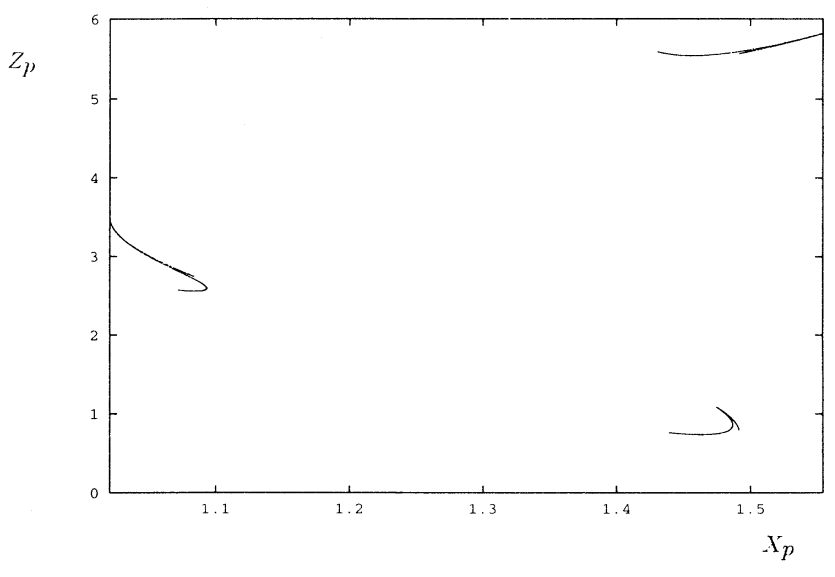

FIG. 6. The Poincare section of the chaotic flow displayed in Fig. 3.

There is an apparent contradiction between the predictions of both flow reconstructions. In one case the period three orbit $B\left(\sigma_{1}, \sigma_{2}\right)=\sigma_{2}^{-1} \sigma_{1}$ implies chaos and in the other case $B\left(\sigma_{1}, \sigma_{2}\right)=\sigma_{1}^{-1} \sigma_{2}^{-1}$ does not.

The contradiction can be resolved as follows.

(1) A periodic orbit $p$, can force a positive topological entropy because of the stretching and bending that its braid type forces upon the complement of the orbit in a disk, $D-p$ [see Fig. 2(d)].

(2) The strange attractor (if it exists) is supported in $D-p$. Qualitative maps of the disk onto the disk are sketched in Figs. 2(c) and 2(d) for the two braids here considered.

(3) In the present case the attractor in the Poincaré section lies in three disconnected pieces and no "experimental" points can be found outside these three pieces. The full attractor is inside a tube braided as the period three orbits (see Fig. 6).

(4) The chaos predicted (or not) by one or the other embedding corresponds to an invariant set that is supported in the part of the disk for which we have no information. Hence, each prediction relies on the hypothesis that the embedding can be extended to the disk. Clearly, both of them cannot be true since we would then have a real contradiction; perhaps none of them can be extended to the full disk.

It is then clear that our prediction of chaos or no chaos depends on our additional hypothesis every time the strange attractor implied is supported in a set disjoint of the sampled set. The moral of the story is that every time we predict a positive topological entropy using topological methods there is a need to verify that the detected strange attractor lies at least partially within a region of phase space well represented in our data set and that the reconstructed manifold can be legitimately considered a deformed disk.

\section{CONCLUSIONS}

We have built an example in which the periodic orbits reconstructed from a scalar time series are characterized by embedding dependent topological numbers. For a period three close return, some embeddings allow us to characterize it as a badly ordered orbit. Other embeddings of the scalar close return give rise to well ordered orbits. This apparent contradiction is rooted in the fact that the full attractor is inside a braided tube, and therefore no complete information is available on the complement of the orbit in a disk.

The example reported in this work provides a negative answer to the following conjecture: "the topological structure of a chaotic flow is embedding independent," at least to the degree to which this example is applicable.

The implication of chaos by badly ordered orbits has been invoked several times in the literature. It is likely that in those cases the embeddings could be extended to whole disks, but this work should raise a red flag on the black-box-like application of topological tools to analyze data.

\section{ACKNOWLEDGMENTS}

This work has been partially funded by Fundacion Antorchas. G. B. M. and H. G. S. are members of CONICET, Argentina.
[1] N. B. Tufillaro, H. G. Solari, and R. Gilmore, Phys. Rev. A 41, 5717 (1990).

[2] G. B. Mindlin, H. G. Solari, R. Gilmore, X. Hou, and N. B. Tufillaro, Phys. Rev. Lett. 64, 2350 (1990).

[3] G. B. Mindlin, H. G. Solari, N. Natiello, R. Gilmore, and X. Hou, J. Nonlinear Sci. 1, 147 (1991).

[4] P. Cvitanovic, G. H. Gunaratne, and I. Procaccia, Phys. Rev. A 38, 1503 (1988).

[5] G. H. Gunaratne, P. S. Lindsay, and M. J. Vinson, Phys. Rev. Lett. 63, 1 (1989).

[6] J. S. Birman and R. F. Williams, Topology 22, 47 (1983).

[7] P. Holmes, in New Directions in Dynamical Systems, edited by $T$. Bedford and J. Swift (Cambridge University Press, Cambridge, England, 1988), p. 47.

[8] F. Papoff, E. Arimondo, F. Fioretto, G. B. Mindlin, H. G. Solari, and R. Gilmore, Phys. Rev. Lett. 68, 1128 (1992).
[9] M. Lefranc and P. Glorieux, Int. J. Bif. and Chaos 3, 643 (1993).

[10] M. Leblanc, P. Glorieux, F. Papoff, F. Molesti, and E. Arimondo, Phys. Rev. Lett. 73, 1364 (1994).

[11] T. Ondarcuhu, G. B. Mindlin, H. L. Mancini, and C. Pérez-García, J. Phys.: Condens. Matter 6, A427 (1994).

[12] T. Ondarcuhu, G. B. Mindlin, H. L. Mancini, and C. Pérez-García, Phys. Rev. Lett. 70, 3892 (1993).

[13] L. H. Kauffman, Knots and Physics (World Scientific, Singapore, 1991).

[14] J. M. Gambaudo, S. Van Strien, and C. Tresser, Ann. Inst. Henri Poincaré A 49, 335 (1989).

[15] H. G. Solari and R. Gilmore, Phys. Rev. A 37, 3097 (1988).

[16] H. Mancini, D. Maza, C. Perez-García, G. B. Mindlin, and M. Huerta (unpublished). 
[17] H. Whitney, Ann. Math. 37, 654 (1936).

[18] N. Packard, J. Crutchfield, and R. Shaw, Phys. Rev. Lett. 45, 712. (1980); F. Takens, Detecting Strange Attractors in Turbulence, Lecture Notes in Mathematics Vol. 898 (Springer-Verlag, Berlin, 1981).
[19] T. Sauer, J. A. Yorke, and M. Casdagli, J. Stat. Mech. 65, 579 (1991).

[20] H. D. I. Abarbanel, Brown, J. J. Sidorowich, and L. Sh. Tsimring, Rev. Mod. Phys. 65, 1331 (1993). 